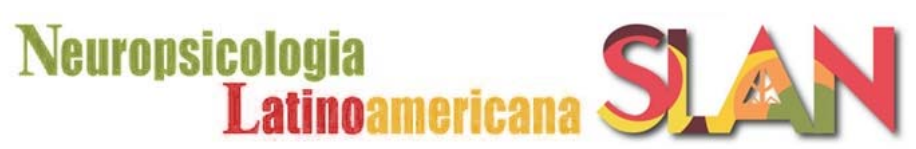

Revista Neuropsicologia Latinoamericana

ISSN 2075-9479 Vol 3. No. 2. 2011, 39-49.

\title{
Rehabilitación neuropsicológica en un caso de afasia semántica
}

\author{
Intervention neuropsychologique dans un cas d'aphasie sémantique \\ Reabilitação neuropsicológica em um caso de afasia semântica \\ Neuropsychological rehabilitation in a case of semantic aphasia
}

\section{Fernando Rodríguez Vargas¹, Yulia Solovievaํㅜ, María del Rosario Bonilla Sánchez¹, Héctor Juan Pelayo González ${ }^{1}$ \& Luis Quintanar Rojas ${ }^{1}$}

\author{
${ }^{1}$ Benemérita Universidad Autónoma de Puebla, Mexico.
}

\section{Resumen}

\begin{abstract}
La afasia se define como una alteración sistémica del lenguaje como consecuencia de lesiones cerebrales. De acuerdo a la propuesta de A.R. Luria, los síndromes afásicos pueden clasificarse según el mecanismo neuropsicológico que se encuentra en la base de las alteraciones que el paciente presenta. En el caso de la afasia semántica la estructura acústica del discurso se encuentra intacta, mientras que el nivel lógico-gramatical se desintegra. Los pacientes con esta forma de afasia presentan alteraciones lingüísticas peculiares: no acceden a las estructuras lógico-gramaticales complejas en el lenguaje oral y escrito con conservación de la repetición de sonidos y palabras. El objetivo de este artículo es presentar el caso de un paciente con síndrome de afasia semántica y mostrar los resultados de la elaboración y la aplicación de un programa de rehabilitación neuropsicológica. Se presenta el contenido del programa de rehabilitación con los ejemplos de ejecuciones de la paciente antes y después de la intervención. Se discute que un programa de rehabilitación neuropsicológica elaborado a partir del análisis preciso del defecto central conduce a los efectos positivos en al actividad verbal y no verbal del paciente, relacionada con análisis y síntesis de las relaciones espaciales.

Palabras-Clave: Traumatismo craneoencefálico; afasia semántica; rehabilitación neuropsicológica.
\end{abstract}

Résumé

L'aphasie se définit comme un désordre acquis du langage. Dans l'approche de A.R. Luria, il est possible de classifier les syndromes aphasiques selon les facteurs neuropsychologiques ou les mécanismes qui déterminent le syndrome observé chez chaque patient. Dans le cas de l'aphasie sémantique, le niveau phonémique du langage est préservé, mais des atteintes sévères sont observées sur le plan de la logique et de la grammaire. Les patients présentant ce type d'aphasie manifestent des troubles singuliers de la production et de la compréhension au niveau de la phrase et du texte. De tels troubles sont dus à l'absence d'accès aux structures grammaticales complexes comprenant des relations temporelles, spatiales ou causales. L'objectif de cet article est de décrire le cas d'une patiente présentant une aphasie sémantique et pour laquelle des stratégies d'évaluation et de traitement neuropsychologique ont été conçues. Le programme d'intervention, par activité et assorti d'exemples concrets, est présenté avec les résultats de l'évaluation initiale et finale. Nous concluons qu'un programme d'intervention neuropsychologique élaboré à partir du diagnostic précis entraine des effets positifs dans les activités verbales et non-verbales de la patiente.

Mots-clefs : Traumatisme cérébral, aphasie sémantique, intervention neuropsychologique.

\section{Resumo}

Afasia é conhecida como uma alteração sistemática da linguagem. Na abordagem de A.R. Luria é possível classificar as syndromes afásicas de acordo com fatores ou mecanismos neuropsicológicos que determinam a síndrome observada em cada paciente. No caso da afasia semântica, o nível fonológico da linguagem está preservado, mas importantes déficits de lógica e de gramática podem ser encontrados. Pacientes com este tipo de afasia manifestam dificuldades peculiares na produção e compreensão linguísticas, nos níveis de sentença e de texto. Tais déficits devem-se à ausência de acesso a estruturas gramaticais complexas com relações temporais, espaciais e causais. O objetivo deste artigo é apresentar um caso de uma paciente com afasia semântica com estratégias de avaliação e de reabilitação neuropsicológicas desenvolvidas para ela. O programa de reabilitação como tarefas e exemplos é apresentado com os resultados das avaliações inicial e final. Conclui-se que um programa de reabilitação neuropsicológica baseado em diagnóstico preciso produziu efeitos positivos em aspectos verbais e não-verbais da paciente.

Palavras-chave: Traumatismo craniano; afasia semântica; reabilitação neuropsicológica.

Artículo recibido: 27/05/2011; Artículo revisado: 15/08/2011; Artículo aceptado: 30/08/2011.

Fernando Rodríguez Vargas, Maestría en Diagnóstico y Rehabilitación Neuropsicológica, Benemérita Universidad Autónoma de Puebla, México. Yulia Solovieva, Maestría en Diagnóstico y Rehabilitación Neuropsicológica, Benemérita Universidad Autónoma de Puebla, México. María del Rosario Bonilla Sánchez, Maestría en Diagnóstico y Rehabilitación Neuropsicológica, Benemérita Universidad Autónoma de Puebla, México. Héctor Juan Pelayo González, Maestría en Diagnóstico y Rehabilitación Neuropsicológica, Benemérita Universidad Autónoma de Puebla, México. Luis Quintanar Rojas, Maestría en Diagnóstico y Rehabilitación Neuropsicológica, Benemérita Universidad Autónoma de Puebla, México.

E-mail: yulia.solovieva@fsic.buap.mx

DOI: $10.5579 / \mathrm{rnl} .2011 .0067$ 


\begin{abstract}
In the case of semantic aphasia the phonological level of language is preserved, but severe logic and grammar deficits can be found. Patients suffering from this type of aphasia manifest peculiar impairments of language production and comprehension at the level of sentences and texts. Such disturbances are due to the absence of access to complex grammar structures with temporal, spatial, and casual relations. The aim of this article is to present a case of a patient with semantic aphasia with the strategies of neuropsychological assessment and rehabilitation designed for her. The rehabilitation program with tasks and examples is presented with the results of the initial and final assessments. We conclude that a program of neuropsychological rehabilitation based on a precise diagnosis produced positive effects in the verbal and nonverbal activity of the patient.

Keywords: Brain injury; semantic aphasia; neuropsychological rehabilitation.
\end{abstract}

A lo largo del tiempo se han propuesto diferentes definiciones acerca de lo que representa el término afasia. Hécaen (1977) la define como un déficit en la comunicación verbal resultante del daño cerebral. Goodglass y Kaplan (1972) consideran a la afasia como perturbaciones del lenguaje oral y escrito que son producidas por lesiones en ciertas áreas cerebrales. Estos mismos autores afirman que no pueden ser consideradas como desórdenes afásicos las alteraciones de articulación de palabras y las alteraciones de la escritura, debidas a la incoordinación o parálisis de los músculos correspondientes, ya que éstas, se deben a la afectación intelectual severa o alteraciones visuales o auditivas. Para Álvarez (2008) la afasia es un trastorno del lenguaje que se caracteriza por la pérdida total o parcial de la capacidad de comprender o utilizar el lenguaje ya formado, lo cual está dado por una dificultad de carácter sensorial o motriz, y que tiene como causa una alteración o lesión orgánica a nivel cortical en las áreas correspondientes a los analizadores auditivo-verbal y verbo- motriz.

Existen distintas clasificaciones de la afasia en la actualidad, en su gran mayoría derivan de las observaciones de Lichtheim-Wernicke. Quintanar (1999) menciona que en algunos tipos de afasia se describe su mecanismo (motora, sensorial, de conducción), en otros tipos de afasia se antepone el defecto primario (motora transcortical), y más aún, en otras se recurre a la base neuroanatómica (sensorial transcortical). Se debe señalar además, que la mayoría de los autores de las diferentes escuelas (francesa, americana, alemana) no designan el mecanismo básico y en su lugar observamos los defectos básicos, y en algunos casos la posible causa del síndrome.

Para nuestro estudio es de suma importancia, mencionar la concepción de afasia según Luria (1947), de acuerdo a la cual la afasia es la alteración sistémica del lenguaje que surge ante lesiones corticales. Esta alteraciones sistémica se manifiesta en algún tipo de síndrome específico, en el cual es posible identificar un mecanismos alterado central o el “defecto primario" Luria (1977). Tsvetkova (1988) refiere que afasia necesariamente implica una desintegración de toda la esfera psíquica del hombre, alterando en primer lugar la función comunicativa del lenguaje.

En el caso de al afasia semántica, el mecanismo central alterado es la síntesis espacial simultánea. Síntesis espacial simultánea se refiere a la posibilidad de orientarse en el espacio en el nivel material, perceptivo y verbal o cuasi espacial, es decir, al reflejo conceptual lógico de las relaciones espaciales. La alteración de este mecanismo conduce al defecto central: la dificultad en la comprensión y producción de las estructuras lógico-gramaticales complejas (Quintanar \& Solovieva, 2002). Lo anterior significa que las dificultades de los pacientes con la afasia semántica no se limitan a dificultades verbales, sino también reflejan en la escritura, lectura, cálculo, actividad constructiva y todas las demás formas de actividades humanas que requieren de análisis y síntesis espaciales simultáneas (Luria, 1977; Luria \& Tsvetkova, 1979). Desde este punto de vista, la afasia no puede considerarse aisladamente de las alteraciones constructivas, preceptivas, escritura, operaciones de cálculo, etc. Es decir, se obtiene un síndrome neuropsicológico particular (Eslava-Cobos \& Cols., 2008). Lo particular de este síndrome es que la estructura acústica del discurso se encuentra intacta Luria (1964). Además, en esta forma de afasia no se percibía la alienación del significado con la palabra en sí misma, como es en el caso de la afasia sensorial. Esto puede conducir al hecho de que la afasia semántica no se detecta durante las evaluaciones de rutina si el evaluador aplica solo pruebas de producción o repetición del lenguaje que no implica análisis y síntesis simultáneas de la información. De hecho, la afasia semántica durante cierto tiempo no se consideraba en las clasificaciones de las afasias (Ardila, 2005; Geschwind, 1965a, b).

La afasia semántica constituye un síndrome complejo en el que se alteran no sólo las formas del lenguaje (oral y escrito), sino todas las esferas de la vida psíquica (cognoscitiva, volitiva, afectivo-emocional y la personalidad) del paciente (Quintanar \& Solovieva, 2002). Es importante considerar que en los casos de daño cerebral, la esfera afectivo-emocional se altera de manera específica, en relación con el estado de otros procesos psicológicos. Por ejemplo, en la afasia se afecta tanto el lenguaje expresivo como impresivo, pero además se alteran también otros procesos psicológicos como la lectura, la escritura, el cálculo, etc. y, en general, se afecta toda la esfera psíquica del hombre que incluye a las emociones y los sentimientos (Quintanar \& Cols., 2001).

En la neuropsicología desarrollada por Luria lo esencial es la cualificación, por lo que la valoración se orienta al análisis de la forma en que se realizan las tareas, en la naturaleza y el tipo de las dificultades y en los apoyos que resultan útiles para la ejecución exitosa de la tarea (Luria, 1977; Glozman, 1999, 2002; Xomskaya, 2002). Todas estas dificultades se establecen a través del análisis cualitativo que permite la caracterización de los errores y la identificación de los mecanismos cerebrales comprometidos. El diagnóstico se establece a través de un proceso complejo de identificación del factor o factores que dificultan o imposibilitan la ejecución del paciente (Quintanar \& Solovieva, 2002). El diagnóstico, finalmente, permite elaborar procedimientos específicos de rehabilitación enfocada a la superación de las alteraciones centrales del síndrome.

El objetivo de nuestro estudio nuestro consistió en la elaboración y aplicación de un programa de rehabilitación 
neuropsicológica en un caso de afasia semántica y mostrar los resultados que se obtienen a partir de trabajo con el paciente.

\section{Método}

\section{Caso clínico}

Se trata de un paciente femenino de 34 años de edad, diestra, de escolaridad secundaria completa, de profesión oficial de policía. La paciente sufrió un traumatismo craneoencefálico severo en región cortical posterior. La presencia del daño cerebral cortical se demuestra a partir de los datos de registro electroencefalográfico cuya interpretación refiere alteración irritativa crónica temporocentral y parietal bilateral de origen cortical. Según el reporte neurológico la paciente presenta perdida del olfato, gusto y presencia de crisis intensas caracterizadas por rigidez generalizadas, las cuales van acompañadas por pérdida de consciencia y control de esfínteres. Actualmente dichas crisis son controladas con medicamento (oxocarbamazepina).

Como consecuencias del trauma craneoencefálico sufrido, la paciente presenta olvidos frecuentes en cuanto a direcciones, anosmia y dificultades en la orientación tiempo y lugar. La recepción de información en su nivel primario está conservada; las áreas primarias están intactas en la recepción de la información en sus diferentes modalidades (visual, auditiva y somatosensorial). La paciente acude al servicio de la Maestría en Diagnóstico y Rehabilitación neuropsicológica de la Facultad de Psicología de la BUAP solicitando apoyo para superar problemas de memoria, lectura y la escritura, así como comprensión del lenguaje.

\section{Procedimiento}

La evaluación neuropsicológica inicial se llevo a cabo en la Unidad Universitaria de Neuropsicología del Hospital Universitario de Puebla. Dicha evaluación se realizo en 5 sesiones individuales de aproximadamente una hora de duración dependiendo del desempeño de la paciente. Posteriormente se realizó un análisis cualitativo de los resultados, lo que permitió identificar el mecanismo central que subyace el síndrome neuropsicológico. Para el análisis cualitativo se consideró el proceso de ejecución de las tareas por parte de la paciente. Realizado dicho análisis, se procedió a la creación y aplicación del programa rehabilitatorio, el cual fue aplicado en dos sesiones cada semana durante un plazo de seis meses con un total de 37 sesiones individuales de 60 minutos de duración. Posterior al programa rehabilitatorio, se realizó una segunda evaluación neuropsicológica, con la que se pudo analizar de manea comparativa el desempeño y ejecución de la paciente antes y después de la aplicación de dicho programa.

\section{Instrumentos Aplicados}

Durante la aplicación se utilizo la Evaluación Neuropsicológica Breve en Español (Neuropsi) (OstroskySolis \& Ardila, 2005), el Esquema de Evaluación del Daño Cerebral (Ostrosky-Solis \& Ardila Alfredo, 1998), Stroop Task (Kaplan, 1935) y la tarea de copia y reproducción de la figura compleja de Rey (1959). La evaluación de la actividad intelectual se realizó a través de la selección de algunas tareas específicas del Protocolo de Evaluación de la Actividad Intelectual (Solovieva et al., 2001, 2002; Solovieva, Chávez, \& Quintanar, 2001), tales como, lectura y análisis de textos narrativos, artísticos y descriptivos, tareas de análisis de

Revista Neuropsicologia Latinoamericana (2011), 3(2), 39-49 cuadros artísticos, tarea de composiciones escritas sobre un tema determiado y un tema propio y la tarea de comprensión de refranes. Dichas tareas se basan en propuestas por Luria (1969), Zeigarnik (1981); Tsvetkova (1996) y Quintanar y Solovieva (2001). Estas tareas se presentan en la Tabla 1.

\section{Resultados de evaluación inicial}

Los resultados obtenidos en la evaluación neuropsicológica inicial mostró la conservación de los mecanismos que garantizan y regulan la comprensión de los sonidos del lenguaje: la paciente discriminó adecuadamente palabras con fonemas opuestos y fonemas cercanos por punto y modo de articulación. Se observó una buena comprensión de palabras concretas - frecuentes y largas - no frecuentes, así como de las órdenes directas - cortas.

En la evaluación de los procesos complejos de síntesis espaciales simultáneas, la paciente se mostró desorientada en las tareas de seguimiento de instrucciones con contenido espacial (señale donde está el perro camina delante del carro y detrás de la señora). En la Figura 1 se puede observar la ejecución durante la tarea "Copia y Reproducción de la Figura de Rey”, en la cual no logro recuperar todos los elementos que la constituyen, así como fallas en la distribución y proporción de la figura. La prueba de modelaje con cubos no fue accesible para la paciente, a pesar de darle muchas ayudas y fragmentar completamente la tarea. La paciente mostró actitud de frustración y disgusto hacía todas las tareas constructivas. Se observaron dificultades para identificar a los animales colocados en diferentes posiciones (de lado y dando la espalda), así como para reconocer a los animales cuando estos están dibujados de forma imprecisa.

La evaluación del lenguaje mostró conservación de producción de fonemas, sílabas y palabras aisladas. Lenguaje espontáneo y directo conservado, así como el lenguaje repetitivo de este mismo nivel. Se evidenciaron dificultades severas en el uso apropiado de preposiciones en construcciones comparativas, genitivas, atributivas, relativas y locativas. La comprensión de las oraciones complejas subordinadas, las cuáles, se construyen con conectivos complejos, fue inaccesible para la paciente. Por ejemplo en la oraciones en ¿Quién es el hermano de la mamá?, ¿Quién es el padre del hermano?, requería de la contextualización para su comprensión. (¿Quién es el hermano de tu mamá?).

En cuanto al trabajo con textos, se observó que cuando son más complejos en su estructura gramatical, se presenta mayor número de errores para acceder al sentido aún cuando la paciente ejercitaba el hábito de lectura antes del trauma (lectura básica), esto es, la paciente consigue acceder al sentido de textos narrativos y descriptivos, pero no de los textos artísticos. Todas estas ejecuciones muestran que la paciente comprende adecuadamente el significado de las palabras aisladas, pero no puede acceder al significado de las construcciones verbales que incluyen giros gramaticales complejos, es decir, las dificultades se observan a nivel de la oración. La Tabla 2 muestra la ejecución de la paciente en el trabajo con textos artísticos.

En la Tabla 3, se muestra la ejecución de la paciente en la tarea de elaboración de planes, en la que se observó una imposibilidad general para organizar el material de manera secuencial. 


\section{REHABILITACIÓN NEUROPSICOLÓGICA Y AFASIA SEMÁNTICA}

Tabla 1

Tareas para la evaluación de la actividad intelectual

\begin{tabular}{|c|c|c|}
\hline \multirow{9}{*}{$\begin{array}{l}\text { Trabajo con } \\
\text { cuadros artísticos }\end{array}$} & TAREAS & CONSIGNA \\
\hline & A) Dos cuadros con el mismo significado y diferente sentido. & 1. ¿Que está representado aquí? \\
\hline & B) Dos cuadros con el mismo sentido y diferente significado. & 2. Describa las emociones de los \\
\hline & $\begin{array}{l}\text { C) Dos cuadros con el mismo sentido y mismo significado pero } \\
\text { visualmente diferentes. }\end{array}$ & personajes del cuadro. \\
\hline & $\begin{array}{l}\text { D) Dos cuadros con diferente sentido y diferente significado } \\
\text { pero visualmente similares. }\end{array}$ & \\
\hline & Trabajo con Textos. & 1. ¿De qué se trata este texto? \\
\hline & A) Texto Narrativos. & 2. Elabore un plan para este texto. \\
\hline & B) Textos Artísticos. & 3. Elabore el título para este \\
\hline & C) Textos Descriptivos. & $\begin{array}{l}\text { texto. } \\
\text { 4. ¿De qué se trata el texto y que } \\
\text { siente usted al leerlo? }\end{array}$ \\
\hline \multirow[t]{2}{*}{ Trabajo con textos } & Composición de un tema dado. & $\begin{array}{l}\text { 1. Elabore un plan para la } \\
\text { composición. } \\
\text { 2. Desarrolle la composición de } \\
\text { acuerdo al plan. }\end{array}$ \\
\hline & Composición de un tema propio. & $\begin{array}{l}\text { 1. Elabore el plan para la } \\
\text { composición. }\end{array}$ \\
\hline \multirow[t]{2}{*}{$\begin{array}{l}\text { Proceso de la } \\
\text { escritura }\end{array}$} & & $\begin{array}{l}\text { 2. Desarrolle la composición de } \\
\text { acuerdo al plan. }\end{array}$ \\
\hline & Comprensión de Refranes. & $\begin{array}{l}\text { Identifique la frase que mejor } \\
\text { refleja el contenido del refrán. }\end{array}$ \\
\hline $\begin{array}{l}\text { Comprensión de } \\
\text { oraciones }\end{array}$ & & \\
\hline
\end{tabular}

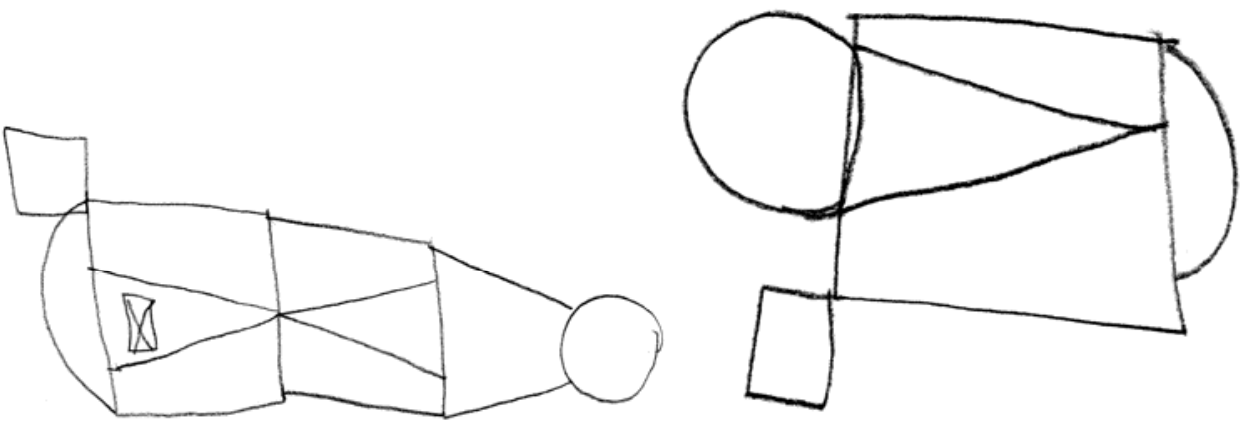

Figura 1. Tarea “Copia y Reproducción de la Figura de Rey”.

Tabla 2

Texto artístico “Lluvia”. Tarea "Trabajo con Textos”

Texto Artístico $\quad$ Evaluación Inicial

¿ De qué se trata este texto y qué siente al leerlo?

La lluvia tiene un vago secreto de ternura, algo de soñolencia resignada y amable, una música humilde se despierta con ella que hace vibrar el alma dormida del paisaje. Es un besar azul que recibe la Tierra, el mito primitivo que vuelve realizarse. El contacto ya frío de cielo y tierra viejos con una mansedumbre de atardecer constante. Es la aurora del fruto. La que nos trae las flores y nos unge de espíritu santo de los mares. La que derrama vida sobre las sementeras y en el alma tristeza de lo que no se sabe. La nostalgia terrible de una vida perdida, el fatal sentimiento de haber nacido tarde, o la ilusión inquieta de una mañana imposible con la inquietud cercana del color de la carne.
"Siento amor, es como una poesía, en esta si me sentí bien, es lo que pasa uno, lo que necesita, lo que no tuvo uno en la niñez al paso de los años lo obtiene, el amor, el cariño, etc.”. 
Tabla 3

Trabajo con textos

Texto “ La Zorra y las Uvas” $\quad \begin{gathered}\text { Evaluación Inicial } \\ \text { "Elabore el plan para este texto”. }\end{gathered}$

Es voz común que a más del mediodía En ayunas la zorra iba cazando; Halla una parra, quedase mirando De la alta vid el fruto que pendía. Causábale mil ansias y congojas No alcanzar a las uvas con la garra Al mostrar a sus dientes la alta parra, Negros racimos entre verdes hojas. Miró, saltó, y anduvo en probaduras Pero vio el imposible ya de fijo; entonces fue como la zorra dijo:

"No las quiero comer: No están maduras". No por eso te muestres impaciente Si se te frustra, Fabio algún intento; Aplica bien al cuento

Y di: No están maduras frescamente.

$$
\frac{1 \text { A zoro }}{\text { La zorra Estaba Fu Ayonas y }}
$$$$
\text { bosco Comida y le costo }
$$$$
\text { Trabajo Pura encontrarla }
$$$$
\text { al final to ha har coutro }
$$$$
\text { y no ha courto }
$$

A wa ror garma

En el trabajo con cuadros artísticos, también se observó un empobrecimiento de ideas, ya que solamente enlistaba algunos elementos incluidos en los cuadros, además de no establecer relaciones entre los mismos. En el trabajo con refranes, al ser frases con un contenido tanto sintáctico como semántico bastante complejos, la paciente no logró abstraerse de la situación concreta representada y generalizarla, ya que sólo es posible con la comprensión directa de las estructuras lógico-gramaticales presentes en dichos refranes. Por ejemplo, para el refrán “No todo lo que brilla es oro”, la paciente elige la opción "El sol brilla como el oro” como opción correcta.

Es posible decir que todas las actividades que requerían de los actos motores en secuencias, tales como, ensambles de rompecabezas, reconstrucción de cubos y la escritura espontánea (Figura 2) se realizaban sin fluidez y con notables dificultades.

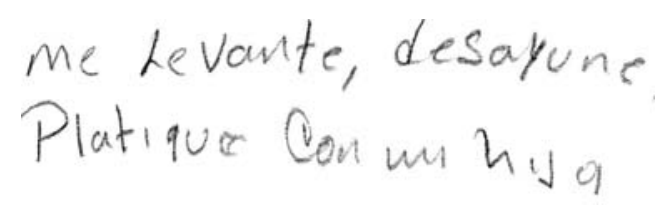

Figura 2. Tarea “Composición de un tema propio”.

En la evaluación de los procesos de regulación y control, se observó que la paciente lleva a cabo todas las tareas y no pierde el motivo de la actividad, sin embargo, requiere de apoyo constante del evaluador en todas las tareas constructivas, perceptivas y verbales. La paciente accede a las orientaciones que le brinda el evaluador.

\section{Programa de Rehabilitación Neuropsicológica}

El programa para la rehabilitación neuropsicológica se conformo de acuerdo a las necesidades de superar las dificultades en análisis y síntesis espaciales simultáneas en el nivel perceptivo y verbal y consecutivamente lograr adecuada comprensión y producción del discurso verbal de la paciente. Dicho programa estuvo estructurado por tres etapas que implicaron distintos grados de dificultad de tareas que incluyen orientación espacial, es decir, las tareas estaban estructuradas con un grado de complejidad creciente, comenzando con aspectos más básicos para así, consecuentemente poder acceder a tareas y procesos más complejos. En cada una de las tareas se construyó la base orientadora de la acción como estrategia de apoyo para el logro o facilitación de las tareas. La tabla 4 muestra las etapas, tareas y algunos ejemplos que se incluyeron dentro del programa rehabilitatorio. 


\section{REHABILITACIÓN NEUROPSICOLÓGICA Y AFASIA SEMÁNTICA}

Tabla 4

Etapas, tareas y ejemplos del programa rehabilitatorio

\section{ETAPA 1}

Objetivo General: Rehabilitar la conciencia de las relaciones espaciales de los objetos y del esquema corporal y la transferencia gradual de dichas operaciones al nivel de discursivo.

\section{TAREAS}

- $\quad$ El paciente debe contestar a preguntas que se le hacen sobre aspectos reflejados en las láminas, o bien que puede deducir de ellas. Se realizan diversos ejercicios trabajando con las expresiones: izquierda, derecha, recto, centro, arriba, abajo, hacia arriba, hacia abajo, delante, detrás, primero, último, dentro, fuera, sale, entra, etc. Se utilizaron las ilustraciones y flechas de apoyo para señalizar la direccionalidad de movimientos y la ubicación de los objetos.

- La paciente debe señalar o colocar determinados signos u objetos (flechas, fichas, figuras geométricas), o bien llevar a cabo órdenes concretas valiéndose de la correspondiente lamina, trabajando especialmente con las construcciones prepositivas y expresiones que se le ofrecen: a, en, bajo, sobre, mas alto, más bajo, ante, tras, de, desde, pon, hacia, etc.

- Mapa/ubicación en espacios conocidos (consultorio, casa, trabajo, país):

- País: Se le otorgan al paciente distintos mapas con creciente grado de dificultad, es decir primero se trabaja con el de la Republica Mexicana, después con el de Estados Unidos, Sudamérica y se finaliza con el de Europa. Se busca que utilizando el mapa. construya oraciones que contengan relaciones espaciales, por ejemplo; “ Puebla está en el centro del país, Chihuahua está arriba de Durango, Estados Unidos está al norte de México, etc.”. Se le pide que las verbalice y que las escriba. Se utilizó la brújula para garantizar adecuada orientación de la paciente en el mapa geográfico.

Ejemplo de ejecución de esta tarea:

$$
\begin{aligned}
& \text { Puabla osta en el Centro del Pais } \\
& \text { Chi duattua esta Arriba de Durango } \\
& \text { Sonora esta a la Izaurerde de chi Hua Hua } \\
& \text { Sinaloa esto abaja de Sonora } \\
& \text { coattuila esta Arriba de Eacatecas } \\
& \text { OHxaca esta al Lado IzQ. de chiapas } \\
& \text { Ouxaca esta al hado Merectto de Gueiverd } \\
& \text { Yucatan esta at haribade Campoche } \\
& \text { Tamavlipas esto abajode Monterrey } \\
& \text { Guan a juato esta bado derecho de Jalisco. } \\
& \text { I stados Unidos es ta al norte de Mexico }
\end{aligned}
$$

- Consultorio, Casa, Oficina: Se le pide que se ubique en el mapa de la ciudad o de la colonia, que trace la ruta de un punto a punto, verbalizando y escribiendo cuales serian las direcciones o pasos a seguir para poder alcanzar dicho punto. Por otro lado también se le podría pedir que dibuje el mapa de su casa, esto con el objetivo de promover la construcción de oraciones con relaciones espaciales por medio de preguntas como "que está a la derecha, que está a la izquierda, que te queda enfrente, etc.”.

- Sistema Solar. Se le presenta un mapa del sistema solar, sobre el cual se le realizan preguntas como “Que está más cerca del sol, la Tierra o Saturno” para posteriormente pedir que la paciente las estructure por si misma y logre verbalizar oraciones como "La Tierra se ubica entre Venus y Marte”, “ La Tierra esta más cerca del Sol que Júpiter”.

- Fósforos: Se le presentan modelos conformados por fósforos, dichos modelos están organizados de tal manera que su complejidad aumenta conforme va superando uno a uno, dicha complejidad se observa tanto en el modelo como en el número de fósforos a utilizar. A la par del armado se le pide que explique y narre las acciones o pasos que está realizando.

- Cubos de Kohs: Se le proporciona al paciente primeramente los modelos a construir en color, para que así este rasgo perceptivo sea de ayuda. Los modelos a construir aumentan de complejidad tanto en el diseño como en el número de cubos a utilizar, comenzando con cuatro y finalizando con nueve. Para cada cubo se propone análisis de los elementos que lo componen con señalación verbal de su mutua ubicación. Se analiza ubicación de cada cuadrante del modelo. Después se aumenta la complejidad de la tarea utilizando los modelos en color negro, es decir, retirando el color rojo el cual le serviría de posible ayuda o guía. A la par del armado se busca que explique el proceso de manera verbal. 
ETAPA 2

Objetivo General: Establecer la comprensión en el nivel de la oración a través del análisis gramatical materializado. TAREAS

- Análisis de oraciones.

- Análisis de textos literarios.

- Composición de un tema propio y dado.

- Trabajo con lenguaje expresivo.

Se le presentaron a la paciente textos de diferente grado de dificultad. Para cada texto se elaboró una guía orientadora de pasos a seguir con ayuda de preguntas. Dichas preguntas se relacionaban con la identificación de elementos sintácticos esenciales de la oración: sujeto y predicado, complemento directo e indirecto. Para cada uno de estos elementos la paciente utilizaba una ficha particular: cuadrado (sujeto), rectángulo (predicado), círculo (complemento directo), círculo pequeño con círculo grande (complemente indirecto). De esta manera, se obtenía el esquema materailziado de la oración con sus elementos esenciales. Se analizaban incisamente oraciones, posteriormente textos. Finalmente se procedió de elaboración de mapas conceptuales (planes) para textos.

\section{ETAPA 3}

Objetivo General: Solución de problemas aritméticos. Para todos los problemas aritméticos se elaboró esquema conceptual que ayuda a identificar la pregunta final, las condiciones, las operaciones, si son suficientes o insuficientes para el logro del objetivo final, así como la dirección imaginaria de las operaciones de suma y multiplicación (derecha) y resta, división (izquierda).

\section{TAREAS}

- $\quad$ Problemas aritméticos simples (una sola operación).

- Problemas simples con estructura gramatical inversa.

- Problemas compuestos (incluyen varias operaciones aritméticas).

- Problemas compuestos e inversos (incluyen varias operaciones aritméticas con estructura gramatical inversa.

- $\quad$ Problemas con una parte desconocida que implican un proceso de búsqueda y orientación mayor.

\section{Resultados Evaluación Neuropsicológica Final}

La evaluación neuropsicológica final, realizada después de la aplicación del programa rehabilitatorio, revelo mejorías en la ejecución de tareas relacionadas con análisis y síntesis espacial.

Las figuras 3, 4 y 5 muestran la ejecución de la paciente en la evaluación final, en tareas como “copia de la casa”, “dibuja un reloj” sin la presencia del modelo y la "figura de Rey”, tanto a la copia como en su reproducción. Se pudo observar una mejoría en la calidad de los trazos, así como en la proporción y distribución de los elementos. De igual manera, se pudo constatar la generación de una estrategia previa a la realización de sus dibujos que logra integrar todos los elementos de manera correcta.

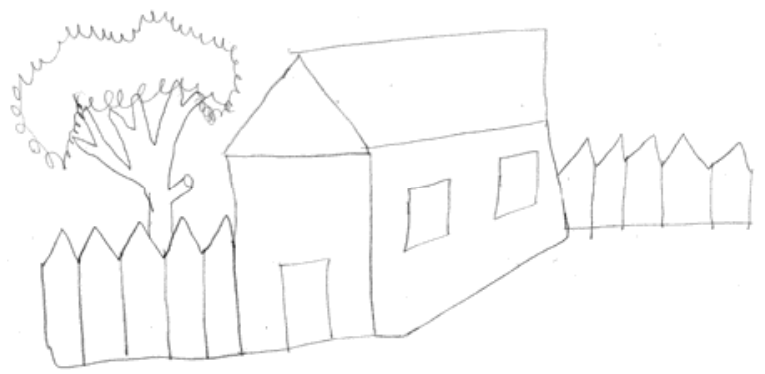

Figura 3. Tarea “Copia de una Casa”.

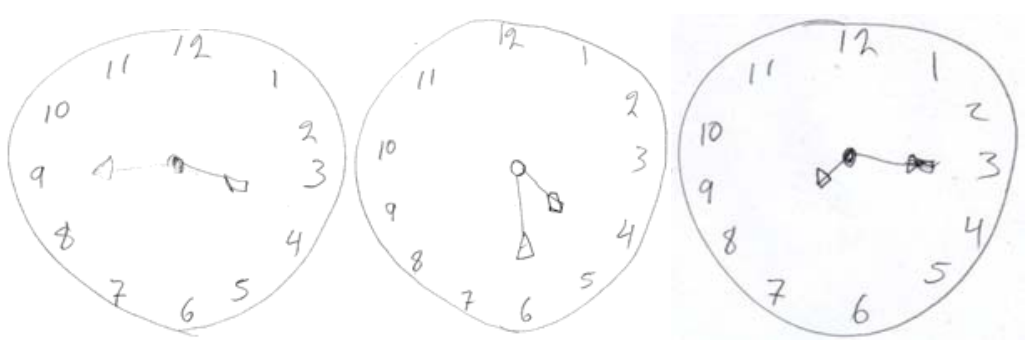

Figura 4. Tarea “Dibuja un Reloj”. 


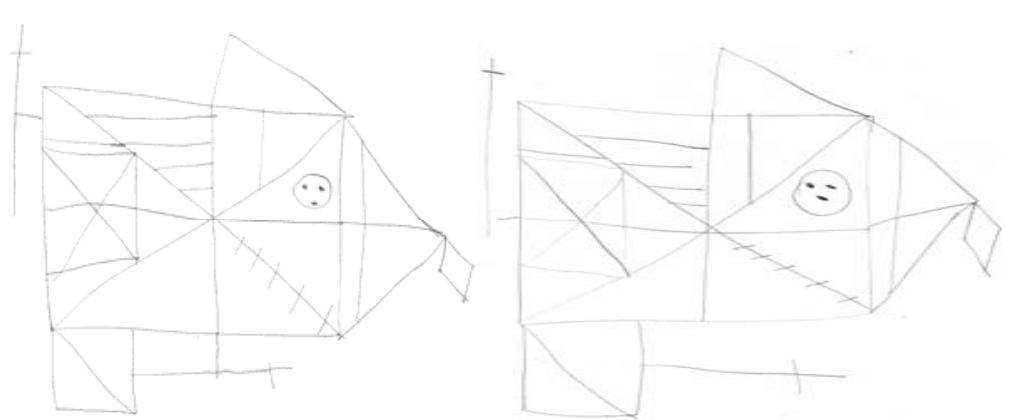

Figura 5. Tarea “Copia y reproducción de la Figura de Rey”.

En la construcción con cubos se observó mejoría definitiva, ya que la paciente logró realizar todos los modelos propuestos por el evaluador. Es importante mencionar que durante la tarea de diseños con cubos, no requirió ayuda de ningún tipo, logrando generar una estrategia previa para el armado, esto es, por medio de la segmentación del modelo. Cabe señalar que la paciente misma reflejó sorpresa y agrado antes la posibilidad de realización de estas tareas, recordando su actitud negativa en la evaluación inicial.

En lo que se refiere a la escritura, es posible constatar mejorías. En la escritura tanto por consigna como espontánea se observó una mayor fluidez y consecución de ideas. Los ejemplos de trabajo con textos evidencian estos logros (Tablas 5 y 6). En los textos se observó mejoría en cuanto al acceso al sentido de los mismos.

Tabla 5

Texto Narrativo "El León y el Ratón”

Evaluación Inicial. $\quad$ Evaluación Final.

¿De qué se trata este texto?

¿De qué se trata este texto?

"De que el ratoncito estaba comiendo su queso y el león “ Aquí trata de que el león encerró al ratón no por queso lo ataco y le grito. Noo, nooo doc, no me acuerdo de sino por el ratón que con sus amigos los estaban nada". molestando, por eso él los encerró, ya que lo encerró, lo dejo libre, y ya de ahí, el león cayó en una trampa, era imposible que saliera de esa red, en eso escuchó el ratoncito que el león estaba rugiendo, cuando escuchó el ratoncito, fue y empezó a desatar la red y dejó libre al león, ósea que ahí agradeció el ratoncito verdad?

Tabla 6

Texto Artístico "Lluvia”

En estos ejemplos se observan mejorías notables en el lenguaje de la paciente que se expresaron en aparición de relaciones comparativas, causales, etc. 
En la figura 6 se observa la ejecución de la paciente al pedirle que elabore tanto el plan como una composición de un tema propio. En la evaluación final se observa un enriquecimiento de ideas, mejor secuencia y estructura en su redacción. En la evaluación inicial dicha ejecución no fue posible.

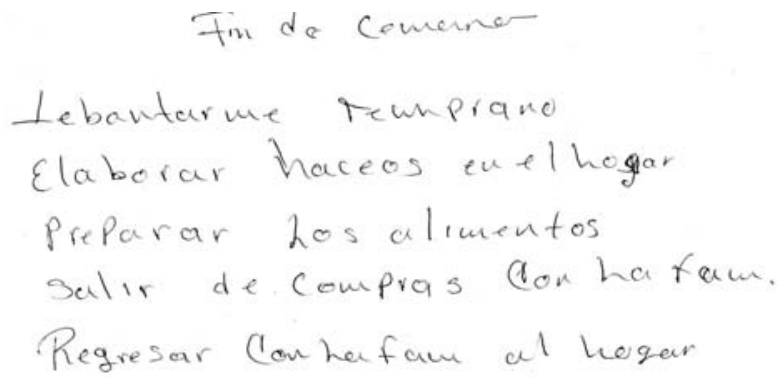

$$
\begin{aligned}
& \text { sab Me hebante Temprano } \\
& \text { Suli. utrabajar. } \\
& \text { fur a bisitar a mi mamer } \\
& \text { Pregrese fuimos de Compras } \\
& \text { pregresmo'àmi cosayprepare ha } \\
& \text { Cena. } \\
& \text { Dom. Me lebante Temprano } \\
& \text { desayone - Para ir ala Igutesio } \\
& \text { Me Prepare Para micasa prepare La comida } \\
& \text { posteriormente Prepare mis cosas Para } \\
& \text { eldia Siguiente - Cene y Lormi. }
\end{aligned}
$$

Figura 6. "Plan y Composición de un tema propio (Fin de Semana)"

En el trabajo con refranes la paciente logró durante la evaluación final abstraerse de la situación concreta representada y generalizarla, esto debido, a que accedió a la comprensión de las estructuras lógico-gramaticales presentes. En esta tarea los errores no se observaron, todas las respuestas fueron correctas.

Independientemente de los logros y mejorías importantes obtenidos en las tareas y ejecuciones contenidas en las diferentes pruebas, es clave hacer mención sobre el impacto que tuvo en la vida diaria de la paciente dichos logros. La paciente se ha vuelto más independiente en su andar cotidiano, ya que logra acudir a su trabajo sin acompañamiento, siempre recordando direcciones y pasos a seguir para localizar su centro de trabajo. También es importante comentar el progreso en cuanto a la comprensión de los textos, que se ve reflejada en aparición del gusto por la lectura diaria de revistas, periódicos, libros, etc. Cabe señalar que antes del inicio de rehabilitación, la lectura no constituía un pasatiempo de preferencia de la paciente. Los familiares han reportado cambios importantes a nivel de orientación, motivación y comportamiento.

\section{Discusión}

Las alteraciones espaciales frecuentemente se citan en la literatura, pero no siempre en relación con al afasia semántica. Por ejemplo, León Carrión (2006) menciona que los pacientes que han sufrido daño cerebral frecuentemente presentan dificultades para orientarse en tiempo y/o espacio; surgen dificultades para saber localizar la información en el tiempo, para saber cuándo ha ocurrido un hecho, dónde se encuentra el paciente, olvido de lugares, personas, la hora, el día, etc. Por su parte, Ardila y Ostrosky (1991) refieren que los pacientes con afasia semántica se distinguen por las dificultades en la comprensión de: estructuras lógico gramaticales que expresan relaciones espaciales, comparativas, espaciotemporales, oraciones pasivas y frases subordinadas y, por último, enunciados en los que intervienen preposiciones que permiten construcciones inversas.

Benson y Ardila (1996) mencionan que la afasia también puede asociarse con cambios intelectuales más generales, no limitados al lenguaje. La afasia nunca debe considerarse como un trastorno que afecta exclusivamente el

Revista Neuropsicologia Latinoamericana (2011), 3(2), 39-49 lenguaje. Todo lo anterior complementando el hecho de que las alteraciones del lenguaje no constituyen cuadros clínicos aislados, sino que forman parte de un síndrome que implica la afectación sistémica de otros procesos psicológicos.

Nuestros resultados concuerdan con lo propuesto por Quintanar (2001) en el aspecto de que las alteraciones del lenguaje, que se observan en los casos de afasia semántica, necesariamente se acompañan por defectos particulares en los procesos intelectuales. Por ejemplo, se presentaron ejecuciones diferenciales de la paciente, en dependencia del texto presentado. Lo anterior se observó durante el trabajar con textos artísticos, los cuales al poseer una estructura más compleja, produjeron mayores dificultades en nuestra paciente en la evaluación inicial. Lo anterior contrasta con el trabajo con textos descriptivos, los cuales incluyen elementos que facilitan su comprensión. Estos elementos son: ausencia de oraciones complejas, subordinadas, orden directo de acontecimientos, presencia de marcadores como nombres de personajes y lugares que aparecen en el orden directo. Estas mismas características de pacientes con la afasia semántica en relación con el trabajo con textos y cuadros artísticos complementan los resultados presentados en estudio previos (Quintanar \& cols., 2001). En este estudio se refiere que las dificultades que surgen durante el trabajo con textos literarios se deben a la imposibilidad para acceder a las estructuras gramaticales que involucran las relaciones espaciales, a veces ocultas, entre los objetos y fenómenos. Cuetos-Vega (2005) menciona que, en casos específicos, a los pacientes se tiene que guiar por el conocimiento temático, por la relación asociativa y la vinculación de los conceptos básicos a lugares o sucesos. El autor refiere que esto se debe a la ausencia de una abstracción mental debida, probablemente, a una lesión que daña las zonas de convergencia encargadas de la formación de conceptos abstractos Para este autor, esos problemas no son de naturaleza lingüística, sino conceptual, como demuestra el hecho de que realicen bien las tareas lingüísticas que no exigen acceder al significado. Obviamente, estos problemas semánticos repercuten en su lenguaje, ya que no se puede apoyar en una teoría del mundo sólida que le sirva de base para su uso lingüístico, dado que las palabras van unidas al significado. Vendrell (2001) comenta que los sujetos afectados por este tipo de afasia presentan un trastorno 
importante de la comprensión con una expresión verbal fluente, muchas veces en forma de jerga semántica. CuetosVerga (2001) menciona que cuando la lesión se localiza en el sistema semántico, el paciente no consigue activar los atributos correspondientes a un concepto determinado y en su lugar selecciona otro término con el que comparte atributos o con el que se relaciona. También asegura que el sistema semántico es común para la comprensión y la producción, de manera que, si un paciente tiene dañado el sistema semántico, también tendrá dificultades y cometerá errores semánticos en comprensión.

Ducarne (1989) asegura que las dificultades de los pacientes con afasia durante el trabajo con textos dependen del grado de complejidad de los constituyentes sintácticos y léxicos, así como de la estructura del discurso en sí, pero no del grado de abstracción de su pensamiento. Se puede decir que en la afasia semántica las dificultades para la identificación de las emociones se relacionaron con la afectación del descifrado de las estructuras lógicogramaticales de los textos (Solovieva et al., 2001). En cuanto al trabajo con refranes, la paciente no lograba abstraerse de la situación concreta representada y generalizarla, debido a esta dificultad con la comprensión de las estructuras lógicogramaticales presentes en dichos refranes. Estos resultados concuerdan con otras publicaciones (Solovieva et al., 2001; Quintanar \& Solovieva, 2002), en las que se señala que las alteraciones en la comprensión de refranes se relacionan con dificultades gramaticales y no con la posibilidad de abstracción. En el trabajo con refranes los pacientes elegían la opción cercana, lo que implica que lograban captar el sentido general del refrán, pero no podían precisarlo ni formularlo a través de la elección precisa de las oraciones. Esto se debe a la imposibilidad para comprender el significado de las construcciones gramaticales como consecuencia de alteraciones de análisis y síntesis espaciales. Estas mismas dificultades se reflejan en el uso apropiado de preposiciones en construcciones comparativas, genitivas, atributivas, relativas y locativas.

Nuestro estudio muestra la importancia del análisis neuropsicológico clínico cualitativo que permite unificar dificultades aparentemente distintas (construcción, cálculo, lectura, lenguaje oral) bajo un único denominador común. En nuestra paciente estas dificultades se relacionaban con olvido de la información, falta en la comprensión lectora, incomprensión del lenguaje oral continuo, problemas en la escritura y las tareas visuo-constructivas. En este caso el factor común es la desintegración de análisis y síntesis espaciales que se refleja en actividades muy diversas. La detección de un único factor que une a estas dificultades permite a su vez relacionar procesos de evaluación, diagnóstico y rehabilitación (Quintanar \& Solovieva, 2001; Solovieva, Bonilla, \& Quintanar, 2008; Quintanar, Solovieva, \& Lázaro, 2009). Esto retoma la importancia de establecimiento de un diagnóstico preciso basado en la detección del defecto central. El análisis a nivel no solo anatómico y cognitivo, sino neuropsicológico cualitativo clínico en casos de cuadros de afasia permite establecer síndromes neuropsicológicos específicos, así como pronosticar el curso y resultados de rehabilitación.

Lo anterior concuerda con el análisis sindromico propuesto por Luria se trata de un análisis dinámico estructural que se integra por las relaciones existentes entre las distintas manifestaciones clínicas (signos y síntomas), análisis que no busca la descripción de alteraciones, sino, la construcción del síndrome, a través de una hipótesis neurodinámica y psicológica que explique el cuadro clínico singular-concreto del paciente.

El programa de rehabilitación fue elaborado en base a la escuela histórico-cultural sobre los principios de rehabilitación propuestos por los seguidores de Luria (Tsvetkova, 1977, 1988, 1996; Quintanar, 1998, 1999). La rehabilitación neuropsicológica basada en la metodología de la escuela histórico-cultural constituye una propuesta novedosa tanto en México como en otros países. Además, no se encuentran publicaciones acerca de rehabilitación de la afasia semántica en pacientes cuyo lengua natal es el español. Nuestro programa fue enriquecido por las propuestas metodológicas desde la teoría de la actividad (Talizina, 2009; Talizina, Solovieva, \& Quintanar, 2010). Consideramos que una de las ventajas de nuestra propuesta de rehabilitación se relaciona con la inclusión de trabajo constante con el elemento esencial de la actividad humana que es la orientación. Así, todas las tareas incluidas en el programa se realizan con apoyo constante en los medios externos, señalizaciones y orientación verbal y objetal del rehabilitador que constituye la base orientadora de las acciones de la paciente. En los pacientes que sufren de este tipo de afasia se desintegra la base orientadora interna de la acción por lo que requieren de constante orientación para la culminación de la tarea (Solovieva, Rentaría \& Quintanar, 2001). En otras tareas como el trabajo con textos y problemas aritméticos, se utilizaron tarjetas de orientación, las cuales contenían los pasos a seguir para poder, en el caso de los trabajos con textos comprenderlo, y en los problemas aritméticos poder descifrar los giros gramaticales que se presentaban y así poder resolver los problemas. Dichos medios externos fueron retirados paulatinamente conforme la paciente internalizaba la base orientadora, para finalmente retirarla y promover la creación de sus propios medios externos de ayuda.

\section{Conclusiones}

1. La relación estrecha entre el proceso de evaluación, diagnóstico y rehabilitación permite establecer el mecanismo neuropsicológico subyacente y de qué forma este mecanismo se manifiesta en las diferentes actividades o esferas en las que se ve inserto el paciente.

2. La utilización del análisis cualitativo resulta fundamental para la conclusión diagnostica.

3. En la afasia semántica, en base de las alteraciones de la actividad intelectual, se encuentra la alteración de análisis y síntesis espaciales, lo cual conduce a las dificultades en la realización de tareas cognitivas diversas.

4. El resultado del trabajo de rehabiltación fue exitoso. No obstante, se espera realizar una evaluación de seguimiento a la paciente para verificar la generalización en el tiempo de las mejorías que fueron identificadas en la evaluación final.

5. El corto periodo del trabajo de rehabilitación con nuestra paciente constituye una de las principales desventajas de la rehabilitación neuropsicológica.

\section{Referencias Bibliográficas}

Alvarez, L., \& Bermudez, A. (2008). Afasias. Logopedia y Foniatría. Ardila, A. (2005). Las afasias. Miami: Florida State University.

Ardila, A. \& Ostrosky, F. (1991). Diagnóstico del Daño Cerebral Enfoque Neuropsicológico. México: Trillas.

Revista Neuropsicologia Latinoamericana (2011), 3(2), 39-49 
Benson, D., \& Ardila, A. (1996). Aphasia: A clinical perspective. New York: Oxford University Press.

Cuetos-Vega, F., \& Castejón, L. (2005). Disociación de la información conceptual y lingüística a partir de un estudio de caso. Revista de Neurología, 41(8), 469-474.

Cuetos-Verga, F. (2001). ¿Porqué cometen errores semánticos los pacientes afásicos?. Revista de Neurología, 32(10), 970974.

Ducarne, R.B. (1989). Reeducación Semiologica de la Afasia. Barcelona: Masson.

Eslava-Cobos, J., \& Mejía, L., \& Quintanar, L., \& Solovieva, Y. (2008). Los Trastornos del Aprendizaje: Perspectivas Neuropsicológicas. Colombia: Magisterio.

Goodglass, H., \& Kaplan E. (1972). The assesment of aphasia and related disorders. Philadelphia: Lea \& Febiger.

Geschwind, N. (1965a). Disconnexion syndromes in animals and man. Brain, 88, 237-294 (Parte II).

Geschwind, N. (1965b). Disconnexion syndromes in animals and man. Brain, 88, 585-644 (Parte III).

Glozman, J. (1999). Quantitative and cualitative integration of Lurian procedures. Neuropsychology Review, 9(1), 23-32.

Glozman, J. (2002). La valoración cuantitativa de los datos de la evaluación neuropsicológica de Luria. Revista Española de Neuropsicología, 4(2-3), 179-196.

Hécaen, H. (1977). Afasias y apraxias. Buenos Aires: Paidos.

Kaplan E. (1935). The Stroop Test. Madrid, TEA Ediciones.

León Carrión, J. (2006). Daño Cerebral. Una Guía para Familas y Terapeutas. España: Delta Publicaciones.

Luria, A. R. (1947). Cerebro y lenguaje (afasia traumática). Barcelona: Fontanella.

Luria, A. R. (1977). Las funciones Corticales Superiores del Hombre. La Habana: Ed. Orbe.

Luria, A. R. (1986). Las funciones Corticales Superiores del Hombre. Moscú: Universidad Estatal de Moscú.

Luria, A.R., \& Tsvetkova L.S. (1979). La Resolución de Problemas y sus alteraciones. Barcelona: Fontanella.

Luria, A. R. (1999). Outline for the neuropsychological examination of patients with local brain lesions. Neuropsychology Review, 9(1), 9-22.

Luria, A.R. (1964). Factors and Forms of Aphasia. Euck \& Maeve. Ciba Foundation.

Ostrosky, F., \& Ardila A. (2005). Evaluación Neuropsicológica Breve en Español (Neuropsi). México: Universidad Nacional Autónoma de México.

Ostrosky, F., \& Ardila A. (1998). Esquema de Evaluación del Daño Cerebral. México: Trillas.

Quintanar , L. (1998). Problemas teóricos y metodológicos de la rehabilitación neuropsicológica. México: Universidad Autónoma de Tlaxcala.

Quintanar, L. (1999). Modelos neuropsicológicos en afasiología. México: Universidad Autónoma de Puebla.

Quintanar, L., \& Solovieva, Y. (2001). Métodos de Rehabilitación en la Neuropsicología del Adulto. México: Universidad Autónoma de Puebla.

Quintanar, L., \& Solovieva, Y. (2002). Análisis Neuropsicológico de las Alteraciones del Lenguaje. Revista de Psicología General y Aplicada, 55(1), 67-87.

Quintanar, L., \& Villegas, N., \& Jiménez, P., \& Orozco, M., \& Solovieva, Y. (2001). Alteraciones de la Esfera AfectivoEmocional en Diferentes Tipos de Afasia. Cuadernos Hispanoamericanos de Psicología, 2(1), 63-74.

Quintanar, L., Lázaro, E., \& Solovieva, Yu. (2009). La rehabilitación neuropsicológica a través de la reorganización de los sistemas funcionales. En E.A. Escotto, M. Pérez \& M.A. Villa (Eds.) Desarrollo y alteraciones del lenguaje, neuropsicología y genética de la inteligencia. México: UNAM, 249-273.

Rey A. (1959). Test de copie et de reproduction du mémoire de figures géometriques compexes. Paris: Centre de Psychologie appliquée.

Revista Neuropsicologia Latinoamericana (2011), 3(2), 39-49
Solovieva, Yu., Chávez, M., \& Quintanar, L. (2001). Alteraciones de la actividad intelectual en los casos de la afasia semántica. Revista Española de Neuropsicología, 3(4), 12-33.

Solovieva, Yu., Villegas, N., Jiménez, P., Orozco, M., \& Quintanar, L. (2001). Alteraciones de la esfera afectivo-emocional en diferentes tipos de afasia. Cuadernos Hispanoamericanos de Psicología, 1 (2), 63-74.

Solovieva, Yu., Rentaría, F., \& Quintanar, L. (2001) Evaluación y rehabilitación de la actividad intelectual en un caso de la afasia semántica. En: Quintanar L. y Solovieva Yu. (Eds.) Métodos de rehabilitación en la neuropsicología del adulto. México: Universidad Autónoma de Puebla, 189224.

Solovieva, Yu., Chávez, M., Planell, G. \& Quintanar, L. (2002). Alteraciones de la actividad intelectual en pacientes con afasia motora aferente”. Revista Latina de Pensamiento y Lenguaje y Neuropsicología Latina, 10(1), 75-93.

Solovieva, Yu., Bonilla, R. \& Quintanar, L. (2008) Aproximación histórico-cultural: intervención en trastornos de aprendizaje. En: Eslava-Cobos J., Mejía L., Quintanar L. y Solovieva Yu. Los trastornos de aprendizaje: perspectivas neuropsicologías. Textos de neuropsicología Latinoamericana. Tomo 1. Colombia: Magisterio, 227-266.

Talizina, N. F. (2009). La teoría de la actividad aplicada a la enseñanza. México: Universidad Autónoma de Puebla.

Talizina, N., Solovieva, Yu., \& Quintanar, L. (2010). La aproximación de la actividad en psicología y su relación con el enfoque histórico-cultural de L.S. Vigotsky. Novedades educativas, 22(230), 4-9.

Tsvetkova, L. (1977). Reeducación del lenguaje, la lectura y la escritura. España: Fontanella.

Tsvetkova, L. (1988). Afasia y enseñanza rehabilitatoria. Moscú: Enseñanza.

Tsvetkova, L. (1996). Cerebro e Intelecto. Moscú: Educación.

Vendrell, J.M. (2001). Las Afasias: semiología y tipos clínicos. Revista de Neurología, 32(10), 980-986.

Xomskaya, E. (2002). El problema de los factores en la neuropsicología. Revista Española de Neuropsicología, 4(2-3), 151-167.

Zeigarnik, B.V. (1981). Psicopatología. Madrid: Akal. 